Currículo sem Fronteiras, v. 20, n. 1, p. 9-22, jan./abr. 2020

\title{
OS PODERES COMPARATIVOS DOS NÚMEROS E O CONHECIMENTO ANTECIPADO DO NÚMERO NA EDUCAÇÃO ${ }^{1}$
}

\author{
Sverker Lindblad \\ University of Gothenburg - Suécia \\ Daniel Pettersson \\ University of Gävle - Suécia \\ Thomas S. Popkewitz \\ University of Wisconsin - Estados Unidos
}

\begin{abstract}
Resumo
O presente artigo traz apontamentos sobre o papel dos números na construção de sujeitos, escolas e políticas educacionais, também sobre o que são e o que deveriam ser. Entendendo os números enquanto construções sociais, um tipo de linguagem socialmente validada a partir de critérios científicos, são levantadas reflexões sobre como os números catalisam reestruturações objetivas e subjetivas nos sistemas educacionais e em seus sujeitos, em perspectiva global, devido às avaliações internacionais em larga escala. Os números possibilitaram novos paradigmas de comparação entre os sistemas escolares nacionais, que deixaram de ser focada em aspectos compreensivos, para enfatizar aspectos competitivos e hierárquicos. As avaliações internacionais em larga escala e os números que delas provêm dão novos sentidos ao que a educação é e o que ela deveria ser.
\end{abstract}

Palavras-chave: Números; Avaliações Internacionais em Larga Escala; Comparação Educacional.

\begin{abstract}
This article presents notes on the role of numbers in the construction of subjects, schools and educational policies, also about what they are and what they should be. Understanding numbers as social constructions, a type of socially validated language based on scientific studies, reflections are raised on how numbers catalyze objective and subjective restructuringin educational systems and in their subjects, in a global perspective, due to large-scale international assessments. Numbers enabled new paradigms of comparison between national school systems, which are no longer focused on comprehensive aspects, to emphasize competitive and hierarchical aspects, such as large-scale international assessmentsand the numbers that they provide give new senses for what education is and what it should be.
\end{abstract}

Keywords: Numbers; Large-scale International Assessments; Educational Comparison 
Este artigo reflete como os números moldam nossa compreensão da educação, sua dinâmica, práticas, operações, objetivos e missões. O foco está nos poderes comparativos dos números - como as diferenças e semelhanças entre tipos de pessoas e performances são construídas por números, ao longo do tempo e por diversos lugares. Os números parecem neutros e precisos, mas, como todos os símbolos (letras, bandeiras, etc.), as relações entre os números e o que eles representam devem ser produzidas e aprendidas socialmente, e as técnicas para traduzir um para o outro (o símbolo de sua representação), como estatísticas, são construídas em sistemas específicos de raciocínio. Os números dizem pouco por eles mesmos, mas, como se tornaram representações poderosas do mundo moderno, mostrados em tabelas, diagramas ou porcentagens, hoje também são altamente incorporados ao que é, e não é de valor e importância.

Os números não são apenas ferramentas para análises, mas também altamente performativos, pois enquadram nossos pensamentos e concepções das coisas. Se houvesse um purgatório moderno, seria um espetáculo de números que se traduziriam em coisas como diagramas e linhas de regressão, mostrando dramaticamente onde estamos e o que esperar do futuro - medo ou esperança. Os números nos fazem ler o mundo em termos de progresso e crises, altos e baixos, diferenças e semelhanças. De onde e como surgem esses poderes dos números - e quais são suas premissas e condições prévias, uma vez que passaram a desempenhar um papel fundamental em avaliações em larga escala e outras formas de políticas e governança baseadas na ciência?

Cada vez mais, a vida em sociedade e as políticas públicas são apresentadas em números por categorias e por espaço e tempo, e por meio disso em termos de gráficos, listas e tabelas comparativas que mostram progressos ou falhas. Tais apresentações tornaram-se parte de esforços para aumentar a transparência e a accountability na produção de conhecimento com base científica.

As categorias e magnitudes expressas por números incorporam representações e relações sociais que nunca são meramente descritivas. Os números incorporam distinções e diferenciações sobre um futuro desejado, em que estes "agem" como uma tecnologia para atualizá-lo. As categorias aparentemente descritivas de desempenho dos alunos, por exemplo, expressam as crianças como tendo "uma natureza” que as avaliações devem “recuperar”. Mas essa mudança de natureza das crianças também estabelece relações dos indivíduos com normas e valores coletivos atribuídos a uma visão da sociedade. Essa construção de um pertencimento coletivo é dada como universal pelo uso de números e seus algoritmos. As esferas social e individual inscritas no conhecimento pelos números também são contestadas: por exemplo, técnica, epistemológica e ontologicamente, como redução da complexidade e das restrições e limites ao se fazer afirmações válidas no raciocínio estatístico, ou ideologicamente, na tradução de questões educacionais para medidas instrumentais e econômicas.

Os números enquanto um “ator” não são menos evidentes no campo da educação. A pesquisa educacional e as avaliações internacionais circulam para definir o que é e o que deve ser o sistema escolar de sucesso. Dado ao desenvolvimento global atual, uma tarefa 
urgente para a pesquisa educacional é analisar a elaboração e o significado das avaliações internacionais em larga escala, seu desenvolvimento e uso em interação com os discursos profissionais e políticos.

Estamos lidando com essas e outras tarefas semelhantes neste artigo como uma contribuição para os discursos atuais sobre educação, conhecimento educacional e a interseção entre ciência e sociedade. Aqui temos a ambição de identificar um conjunto de problemáticas do conhecimento em contextos educacionais e esclarecer as implicações do conhecimento pelo número na educação.

Como entendemos os números como uma problemática do conhecimento? Números, como Hacking (1983) argumenta, são partes de um sistema de comunicação com tecnologias, criando uma distância dos fenômenos, por parecer resumir eventos e transações complexas (cf. Porter, 1995). A produção e o uso de números são vitais para o governar, assim como a própria governamentalidade (ROSE, 1999). Um aspecto específico dessa problemática é categorizar práticas em estatística (POPKEWITZ; LINDBLAD, 2001) e processos de looping (HACKING, 1992) na criação de tipos humanos por meio de categorização e reflexividade. Números rigorosos e uniformes também têm suas vantagens em permitir o transporte no tempo e no espaço, sem exigir conhecimento íntimo e confiança pessoal, pois parecem excluir críticas na luta conjunta da ciência e das políticas contra a subjetividade.

Os números foram entrelaçados no projeto de modernidade e democracia como garantias de “justiça”, “precisão” e “imparcialidade”. Uma posição central no projeto de modernidade e democracia é ocupada pela educação e todas as expectativas da sociedade inscritas na educação. Essas expectativas podem ser resumidas na educação como um requisito central para o desenvolvimento individual, social e econômico. Em tempos de dominação de um paradigma comparativista, baseado em medidas de classificação e eficiência, em vez da compreensão das culturas educacionais e as formas de lidar com problemas da educação, as comparações internacionais passam a ser vistas como ferramentas importantes para alçar melhorias educacionais e sociais. Um exemplo contemporâneo disso é observado na declaração da Organização para a Cooperação e Desenvolvimento Econômico (OCDE): “É somente através de [...] comparações que os países podem compreender os pontos fortes e fracos de seus sistemas educacionais e identificar as melhores práticas e os caminhos a seguir” (OECD, 2006, p. 18). Isso exemplifica o significado atribuído às comparações como uma ferramenta para a padronização e legitimidade dos sistemas nacionais de educação (cf. KAMENS, 2009; STEINER-KHAMSI; WALDOW, 2012).

Enquanto o paradigma comparativo é evidente na formação das ciências sociais no século XIX (POPKEWITZ, 2008), nesta era de um paradigma comparativista em expansão, as organizações internacionais passam a funcionar como containers do conhecimento, além de produzir novos conhecimentos baseados em análises estatísticas. Nesse contexto, Carvalho (2012) afirmou que as organizações internacionais passam a funcionar como mediadoras do intercâmbio de conhecimento e criam e reformulam ideias e programas, 
fabricando, assim, um instrumento específico de política do conhecimento. Em particular, as organizações que produzem avaliações internacionais em larga escala e pesquisas baseadas nesses tipos de dados tornaram-se um instrumento poderoso para a mudança, padronização e legitimação dos sistemas nacionais de educação.

\section{Números para uma compreensão educacional}

A Associação Internacional para Avaliação de Desempenho Educacional (ADE) foi uma das primeiras organizações a se concentrar em avaliações em larga escala do desempenho individual dos alunos. A Associação foi criada para realizar estudos educacionais comparativos no final da década de 1950 - conduzindo sua primeira avaliação no início da década de 1960 (PETTERSSON, 2014). O primeiro estudo realizado pela ADE diferiu de outros estudos comparativos de educação realizados na época, na tentativa de introduzir uma abordagem empírica baseada em números, em um campo então dominado por análises culturais (FOSHAY Et al., 1962).

Antes da realização do primeiro estudo da ADE, comparar a educação se dava a partir de ideais mais humanísticos, contudo, com a formação da ADE por cientistas interessados em psicometria e com um interesse declarado em resultados educacionais, nas ciências sociais e nas ciências comportamentais, os testes de desempenho passaram a ser o método ideal para comparar a educação (cf. KAZAMIAS; MASSIALAS, 1982). Em 1935, um livro desconhecido escrito pelo francês Marc-Antoine Jullien em 1817 foi doado ao Bureau Internacional de Educação (IBE - Genebra, Suíça). O livro foi lido por Pedro Roselló, que então trabalhava no Bureau. Em 1943, Roselló publicou um texto que apresentava Jullien como o pai da educação comparada (ROSELLÓ, 1943). O que Jullien tentou fazer em seu livro do início do século XIX foi introduzir o positivismo como base para todos os estudos comparativos. Dessa forma, os números se tornaram os fatos objetivos que precisavam ser coletados para permitir reivindicações e cobranças na área educacional.

Em relação ao primeiro estudo da ADE, foi possível, mesmo que o estudo fosse algo novo, reivindicar uma longa história de realização de estudos comparados com base em números. Com isso, a ADE poderia defender uma legitimidade histórica do estudo, que passou a ser importante nas décadas de 1950 e 1960, especialmente no contexto americano de pesquisa em que o positivismo ganhou importância (cf. ANDERSON, 1961). A afirmação de que Jullien foi o pai fundador da educação comparada é algo mais contestado nesse campo (por exemplo, GARCÍA GARRIDO, 1996; NOAH E ECKSTEIN, 1969) e ainda hoje existe uma discussão vívida no campo da educação comparada sobre suas raízes e trajetórias históricas (EPSTEIN, 2008). No entanto, o pensamento de Jullien foi usado para legitimar um estudo, como o conduzido pela ADE, como um esforço estruturado sobre os pilares da ciência e que serviu para dar ao estudo história e legitimidade.

Outra maneira de encarar essa problemática é afirmar que a ADE criou algo novo na história da educação comparada, já que se concentrou no resultado educacional que poderia 
ser representado em números: criou hierarquias de estudantes e sistemas educacionais, bem como de nações com base nesses números e, como resultado, um raciocínio positivista específico sobre educação. Ela continuou a conduzir várias avaliações, como o Trends in International Mathematics and Science Study (TIMSS), o Civic Education Study (CIVED) e o International Civic and Citizenship Education Study (ICCS). Eventualmente, outro ator importante passou a se envolver no campo - a OCDE. A partir do início do século XXI, a OCDE, com a assistência do estudo do Programa Internacional de Avaliação de Alunos (PISA), tornou-se um dos atores mais importantes na realização de avaliações em larga escala e mais organizações passaram a seguir o mesmo caminho.

Esse desenvolvimento, exemplificado pelo PISA, foi resultado de um conflito internacional a partir da década de 1980, de acordo com Cusso e D’Amico (2005). Eles mostram que, nas estatísticas educacionais internacionais, a UNESCO, que na época era a principal produtora de estatísticas, foi criticada pelo Banco Mundial e pela OCDE, que exigiram mais classificações hierárquicas e informações sobre o desempenho dos estudantes. CUSSO e D’AMICO (2005, p.200) afirmam:

Tornou-se necessário não apenas saber quantos graduados um sistema educacional produziu em comparação com os de outros países, mas também a que custo, com quais objetivos e que conhecimentos e habilidades exatas foram adquiridos pelos alunos.

O que encontramos aqui é uma mudança de ênfase nas comparações internacionais. O que importa são comparações de sistemas de ensino que apresentam hierarquias e classificações em desempenho e eficiência. Isso contrasta com comparações internacionais focadas em uma compreensão cultural da educação e como as tarefas e os problemas educacionais são tratados em diferentes contextos. Estamos aqui fazendo uma distinção entre análises comparativas, enfatizando qualidades educacionais e compreensão de questões educativas, e análises comparativísticas, cujo foco está em medidas de classificação e hierarquização. Embora os estudos comparativos internacionais geralmente tenham características comparativas e comparativísticas, o argumento apresentado aqui é que atualmente as análises comparativísticas são mais importantes e que, desde a década de 1990, tornou-se predominante um paradigma comparativístico. Nesse paradigma, a educação pelos números é dominante. Contudo o ponto principal é a hierarquização da educação -o que é melhor e o que é pior - de acordo com os padrões ou critérios usados.

Diante disso, o que realmente está acontecendo quando a educação é transformada em algo possível de se demonstrar em números, e que tipos de pessoas, hierarquias e tipologias são criados? Como exemplo disso, podemos começar analisando um dos apontamentos do PISA sobre o bem-estar dos alunos, divulgado em abril de 2017. O estudo é apresentado nas seguintes palavras: 
Os alunos estão felizes na escola? Eles têm boas relações com seus colegas, professores e pais? Existe alguma ligação entre a qualidade do relacionamento dos alunos dentro e fora da escola e o desempenho acadêmico? Essas perguntas são fundamentais para o 'Students' WellBeing: PISA Results 2015 (volume 3)', o primeiro relatório do PISA/OCDE que analisa o desempenho dos alunos na escola, seus relacionamentos com colegas e professores, sua vida em casa e como eles gastam seu tempo fora da escola. ${ }^{2}$

Em outro relatório do PISA, os argumentos para estudar o desempenho dos alunos e destacar as escolas como elementos importantes continuam nas seguintes linhas:

As escolas não são apenas lugares onde os alunos adquirem habilidades acadêmicas; elas também ajudam os alunos a se tornarem mais resilientes diante das adversidades, a se sentirem mais conectados com as pessoas ao seu redor e a almejarem maiores aspirações para o futuro. Além disso, as escolas são o primeiro lugar em que as crianças experimentam a sociedade em todas as suas facetas, e essas experiências podem ter uma influência profunda nas atitudes e no comportamento dos alunos ao longo da vida. (OECD, 2017; p. 3).

As citações anteriores de avaliações recentes da OCDE parecem não apenas razoáveis, mas, de fato, necessárias. A educação moderna "faz" o sucesso dos alunos nas escolas ligado à felicidade e segurança psicológica, em um mundo que parece continuamente apresentar novos desafios para a juventude. A vida pessoal dos alunos, sentimentos de satisfação e relações com os pais, colegas e sociedade são considerados indicadores de desempenho e caminhos para o sucesso individual na sociedade, bem como a saúde da sociedade na preparação para o futuro. O esforço da OCDE no novo estudo sobre o bemestar dos alunos parece ser uma extensão provável de como a escolaridade é entendida enquanto promotora dos múltiplos objetivos de possibilitar o sentimento individual de satisfação, proporcionar o aproveitamento dos alunos e promover a prosperidade nacional.

Na literatura sobre avaliações em larga escala, esse tipo de raciocínio ordena o que é dito e feito através de sua medição. As inscrições, o gerenciamento e as orquestrações de gráficos, estatísticas e tabelas mapeiam uma racionalidade ou um sistema de razões para dizer a verdade sobre organizações, instituições e pessoas. Os dados de taxonomias populacionais de gênero, distinções socioeconômicas, relacionadas com as classificações dos designs escolares e sistemas educacionais, juntamente com avaliações de desempenho escolar ou outros tipos de medidas incorporam déficits e esperanças como diretrizes para a ação profissional ou política que nunca é meramente descritiva, mas antecipatória.

Por exemplo, a expressão do "bem-estar do aluno” não é apenas uma afirmação descritiva sobre quem a criança é, mas também sobre quem potencialmenteela deveria ser. 
Essa produção é incorporada em tipos de raciocínio sobre "a natureza da criança", considerando o que medir e classificar, bem como quais conclusões são possíveis de tirar e como agir. A pesquisa incorpora dispositivos de inscrição sobre tipos de pessoas que aparecem como qualidades universais que contribuem para o desempenho escolar das crianças em diferentes contextos, que simultaneamente articulam limites normativos sobre o que importa - e deve importar - e fatos para as pessoas agirem. Essa coprodução da pesquisa e da sociedade no setor educacional (ver, por exemplo, NOWOTNY, SCOTT, GIBBONS, 2003; KULLENBERG, 2012), paradoxalmente, busca atualizar o que é expresso como descrição - a fabricação da individualidade e da sociedade que deve introduzir o futuro como deve ser.

Essa configuração do conhecimento sobre o que é antecipatório é expressa em tabelas (ver OECD, 2015, p. 39)nas quais se incorpora um tipo particular de racionalidade calculativa e "objetividade comunicativa" (HALPERN, 2014), que reformulam os princípios epistemológicos das ciências sociais e políticas e o campo ou ágora da política na educação, embora essa reformulação tenha implicações para a circulação da expertise. Os países participantes são comparados e classificados por medidas de satisfação de vida dos estudantes em relação às diferenças de gênero e socioeconômicas, além do desempenho escolar. Dentro de um paradigma comparativístico, observamos que no México há a maior parcela de estudantes satisfeitos com suas vidas, enquanto os coreanos são considerados como os menos satisfeitos.

As qualidades normativas expressas na avaliação internacional da escolarização dos estudantes não são o que se pensa convencionalmente da relação filosófica do "é" e "deveria ser”, assim como também não são as suposições sobre saúde, normalidade e os fatos futuros derivados empiricamente. Essa produção de declarações sobre educação e escolaridade é construída em tipos de raciocínio sobre "a natureza da criança" e éperformada em redes de atores.

\section{A emergência de um raciocínio}

A ideia das ciências sociais incorporando futuros desejados a serem atualizados através do número já era visível no Iluminismo europeu a partir do final do século XVIII. Se olharmos para David Hume e os teóricos britânicos da riqueza, sociedade e economia política no início do século XVIII, a filosofia naturalista considera um mundo desejado de equidade e equivalência como menos relacionado com questões do conhecimento do que sobre a justiça na lei que rege as trocas de mercado. Os números não tiveram papel relevante nos processos de delimitação da justiça e da equidade. A riqueza das nações (1776), de Adam Smith, em contraste com Hume, reformula os cálculos sobre os mercados em questões da ciência como "mercados" padronizados de uma maneira que possa ser projetada em semelhanças com o futuro. O mercado, teoricamente o coração da economia moral de Smith, era um agente para aumentar a riqueza nacional através da "mão invisível” 
dos motivos humanos e da concorrência. Smith inventou maneiras de obter representação numérica através da criação de formas de "medir e calcular como se elas existissem para dizer algo sobre riqueza e governo” (POOVEY, 1998; p. 240-241). Os números não eram descritivos, mas "incorporavam suposições a priori [de Smith] sobre o que o sistema de mercado deveria ser" (POOVEY, 1998; p. 216, itálico no original).

As ciências da sociedade e da riqueza de Smith podem ser entendidas como incorporações do movimento de reivindicações filosóficas sistêmicas sobre o que é considerado universal (natureza humana), para descrições de abstrações (o sistema de mercado) e depois para as quantificações,as quais foram dos efeitos ou produtos das abstrações (trabalho, prosperidade nacional). Os últimos se tornaram os "fatos sociais" que permitem comparações (POOVEY, 1998; p. 237). As operações filosóficas de abstrair e generalizar mercados inscreveram a esperança do filósofo de que seu conhecimento levasse à ação e "se a ação fosse diligentemente perseguida, poderia atualizar o futuro com o qual o filósofo foi o primeiro a sonhar” (POOVEY, 1998; p. 247).

As quantificações como descrições da teoria se tornaram um agente histórico da "natureza humana”, um universal filosófico que poderia ser nomeado e capaz de quantificar os efeitos da abstração. Construir agregados era "registrar a significância desses fenômenos que só poderiam ser conhecidos em retrospecto e descontar o que divergia do tipo, a fim de descrever a 'natureza'” (POOVEY, 1998; p. 226). O esquema histórico deu importância à domesticidade, conduta social, mulheres e sociedade comercial como:

a encarnação mais sofisticada da sociabilidade humana através da qual a mente humana seria revelada coletivamente [. . .] As abstrações de segunda ordem, como trabalho e felicidade, que não eram mais uma reivindicação universal, mas um lugar não retórico (não convincente) para um tipo de representação que descrevia o que poderia ser, como se esse potencial estivesse simplesmente esperando para se materializar (POOVEY, 1998; p. 248, itálico no original).

Nós nos concentramos na ciência da riqueza de Smith, pois fornece uma maneira de pensar historicamente na "sociedade" não como um domínio, mas enquanto algo que é agregado como uma maneira de falar e agir sobre pessoas e de pertencimento coletivo. Antes do século XVIII, na Europa, por exemplo, "sociedade" era uma palavra para descrever uma associação ou grupo de pessoas. A sociedade no final do século XVIII passou a se referir a forças e estruturas anônimas que deram organização à vida humana. $\mathrm{O}$ surgimento da individualidade com uma existência independente paradoxalmente se referia a princípios sobre a sociedade. A noção de Adam Smith da mão invisível dos mercados deu foco às forças abstratas através das quais o indivíduo buscava o interesse próprio na promoção do bem da sociedade. A teoria política de John Locke incorporou princípios sobre a consciência do self ao conhecimento adquirido através das experiências da sociedade. A noção de Jean-Jacques Rousseau do contrato social relacionou o governo e a 
individualidade como elementos centrais na determinação da "vontade geral". Embora diferentes na relação social e individual, os dois não se opõem, mas estão incorporados no mesmo fenômeno.

A inscrição do "social” deu referência às relações abstratas através das quais a individualidade estava ligada às noções iluministas do que é cosmopolita e, posteriormente, foi introduzido nas teorias políticas do cidadão e do coletivo pertencente às novas repúblicas. As noções de inspiração luterana de Gemeinschaft (baseadas na interação pessoal) e Gesellschaft (baseadas na interação indireta), criadas pelo sociólogo alemão Tönnies (1887/2007), deveriam ser, por exemplo, para considerar como as sociedades urbanas poderiam restabelecer suas anteriores comunidades pastorais de pertencimento. As distinções de Gemeinschaft (pastoral) e Gesellschaft (sociedade urbana) viajaram e foram traduzidas para narrativas de salvação calvinistas americanas sobre comunidades pastorais rurais para lidar com o distúrbio moral percebido das populações urbanas do início do século XX. Os conceitos sobre grupos primários ${ }^{3}$ e interacionismo simbólico geraram princípios sobre como o pertencimento social e o "lar" podem ser estabelecidos nas condições e qualidades abstratas e anônimas da "sociedade" industrial e urbana.

Essa formação da sociedade, normas coletivas e valores de pertencimento estão inscritos nas avaliações internacionais por meio de sua expertise de quantificação. A criação desses dispositivos de inscrição sobre as escolas não é apenas sobre o desempenho das crianças, mas também historicamente incorpora princípios sobre "a natureza das pessoas” enquanto sociedade. Porter (2012), por exemplo, destaca que a elaboração de descrições simples é baseada em redes e processos espessos que não podem ser assumidos quando se olha para as tabelas, quadros e gráficos sobre nações e desempenho escolar. Kullenberg (2012) analisa a produção de pesquisas e examina como é possível fazer uma quantificação da sociedade. Ele sugere que o uso de fatos quantitativos sobre uma sociedade molda o que se torna o problema dentro dessa mesma construção do social. Metodologicamente, não há necessidade de interpretação. A produção comum do "social" não exige um desvio através do "significante" ou "discurso". É possível, desde que o material empírico possa ser montado adequadamente, seguir referências a textos, instrumentos, escalas, práticas, pessoas e assim por diante, em um sentido muito concreto.

As inscrições de números são um método de pensamento, uma grade de análise cultural e sociológica, uma imaginação e um método de governo que se move para fenômenos não econômicos, com foco em atributos e correlações que referenciam o comportamento, em vez do processo (Foucault, 2008; p. 218). As avaliações internacionais atuais incorporam teorias sobre individualidade e sociedade nas categorias inscritas nas estatísticas comparativas de avaliação. Na verdade, essas categorias e a construção de equivalências por meio da aplicação de estatísticas não são sobre avaliar o que é, mas sobre o que será. Os números são práticas culturais que incorporam mudanças nas condições sociais e que também mudam as pessoas.

A uniformidade dada pelos números traz ordens pouco comuns na vida social para um sistema de magnitudes que regularizam as relações entre componentes sociais e 
psicológicos (ROSE, 1999). Se nós usarmos a política contemporânea e pesquisarmos sobre pobreza, por exemplo, no Reino Unido e nos Estados Unidos, os números estabelecem categorias de equivalência e as correlacionam para identificar fatores sobre a "unidade" familiar, como características físicas, sociais e psicológicas do lar e das relações parentais. Ao correlacionar as magnitudes estatísticas dessas características populacionais com os níveis de desempenho das crianças, acredita-se que uma sociedade mais igualitária e democrática possa ser alcançada.

Os números funcionam como tecnologias para mapear limites e as características internas dos espaços a serem gerenciados, como estratégia para fazer julgamentos fora do subjetivo. No entanto, enquanto os números “agem” como reais, eles incorporam escolhas implícitas sobre "o que medir, como medir, com que frequência medir e como apresentar e interpretar os resultados” (ROSE, 1999; p. 199).

\section{Números como dispositivos de inscrição}

O pensamento dos números como “dispositivos de inscrição” é importante ao visualizar avaliações internacionais e sua cultura visual, na qual os gráficos, tabelas e quadros são tomados como um modo de dizer a verdade. A coleta e agregação de números participam de uma "limpeza" ou espaço onde o pensamento e a ação podem ocorrer (Rose, 1999; p. 212). Esse espaço cultural na escola implica a fabricação de tipos humanos. A fabricação se concentra nas distinções e classificações sobre as pessoas como tendo qualidades duplas e simultâneas. As categorias sobre crianças e as disciplinas escolares nas avaliações são ficções. O que constitui o ensino de matemática, física e literatura nas avaliações se baseia nos modos de representação e processos de pensamento sobre o aprendizado das crianças encontrado em modelos históricos de currículo da Europa e América do Norte. Isso funciona como os “bastidores” ou reunião de diferentes conjuntos de princípios que conectam e tornam razoáveis os modelos de currículo através dos quais as avaliações são formadas.

Se usarmos a analogia da alquimia, as avaliações serão baseadas em uma variedade de atividades diferentes que ocorrem antes que as avaliações sejam, de fato, construídas por meio de práticas de medição (POPKEWITZ, 1984; 2008). Os modelos curriculares são traduções e transmogrificações ${ }^{4}$ das relações históricas que formam os campos disciplinares das disciplinas escolares. A tradução em modelos curriculares é necessária, pois as escolas têm diferentes conjuntos de relações culturais e padrões de comunicação, entre outros, e os campos disciplinares da física ou da matemática também. Esses modelos escolares de desenho curricular são desenhados e moldados através de teorias psicológicas e sociológicas que foram inventadas para entender a ciência, a história ou a matemática como campos de produção de conhecimento. As psicologias da aprendizagem foram constituídas para governar a ordem moral na qual as crianças se desenvolvem de maneira a antecipar uma idade adulta desejada (ver LESKO, 2001). 
Essa ficção sobre quem a criança é e quem deveria ser se expressa na noção psicológica da adolescência ${ }^{5}$ dos estudos americanos sobre crianças no início do século $\mathrm{XX}$. As psicologias foram inventadas para mudar as maneiras pelas quais as qualidades internas e os valores morais das novas populações estavam entrando na escola de massa particularmente crianças e famílias urbanas, imigrantes e "não brancos” (POPKEWITZ, 2008).

As abstrações são quimeras de primeira ordem: o que são dadas como disciplinas escolares a serem avaliadas são alquimias e quimeras. Se examinarmos o relatório contemporâneo da McKinsey sobre "juventude" (MOURSHED et al., 2014) e o recente relatório da OCDE sobre o "bem-estar" da criança, as classificações e distinções dos estudos sobre infância são formadas sem o reconhecimento dos "bastidores" nos modelos curriculares e nas psicologias que estruturam o que é avaliado. As práticas culturais incorporadas nos números são elididas. A juventude é dada como uma representação e uma identidade particulares para ordenar e organizar o estudo da escolaridade, crime, família e comunidade, entre outros. Sua inscrição como espaço independente modela tecnologias que produzem tipos de pessoas, atuam em populações específicas e funcionam como teses culturais para as pessoas agirem por si mesmas. Nesse sentido, as avaliações internacionais em larga escala (International Large-Scale Assessments - ILSA) são atores produzindo relações de individualidade e sociais que são não apenas descritivas, mas antecipatórias do que deveria ser.

\section{Notas}

1. Artigo traduzido por Lucas Felicetti Rezende e Heloisa Silva de Oliveira Gomes.

2. “First OECD PISA report on Students' Well-Being launches....” 19 abr.. 2017. Disponível em: https://www.oecd.org/newsroom/first-oecd-pisa-report-on-students-well-being-launches-wednesday-19-april2017.htm. Acessado em 19 fev. 2020.

3. O conceito de "grupo primário" de Charles Horton Cooley (2009), um dos membros fundadores da Associação Americana de Sociologia, e o interacionismo simbólico de George Herbert Mead (1934) deram expressão à agência humana dentro da grade de práticas associadas às discussões sobre as condições sociais da vida urbana americana.

4. Mudar ou transformar a aparência ou a forma de algo, geralmente com a consequência de que as características são diferentes. (Nota dos tradutores).

5. “Adolescência” era uma palavra que existia antes de Stanley Hall, mas foi trazida para um domínio das novas ciências da psicologia para racionalizar, classificar e administrar crianças.

\section{Referências}

ANDERSON, C. A. Methodology of comparative education. International Review of Education, 7(1), 123, 1961.

CARVALHO, L. M. The fabrications and travels of a knowledge-policy instrument. European Educational Research Journal, 11(2), 172-188, 2012. 
COOLEY, C. H. Social organization: A study of the larger mind. Ithaca, NY: Cornell University Press, 2009.

CUSSO, R. E D’AMICO, S. From development comparatism to globalization comparativism: Towards more normative international education statistics May 2005. Comparative Education, 41(2):199-216, 2005.

EPSTEIN, E. H. Setting the normative boundaries: Crucial epistemological benchmarks in comparative education. Comparative Education, 44(4), 372-386, 2008.

FOSHAY, A. W.; THORNDIKE, R. L.; HOTYAT, F.; PIDGEON, D. A.; WALKER, D. A. Educational achievements of 13 years old in twelve countries. Hamburg: UNESCO Institute of Education, 1962.

FOUCAULT, M. The birth of biopolitics: Lectures at the Collège de France, 1978-1979. New York: Palgrave Macmillan, 2008.

GARCÍA GARRIDO, J. L. Fundamentos de Educación Comparada (Editorial). Madrid: Dykinson S. L, 1996.

HACKING, I. Representing and intervening: Introductory topics in the philosophy of natural science. Cambridge: Cambridge University Press, 1983.

HACKING, I. "Style" for historians and philosophers. Studies in History and Philosophy of Science Part A, 23(1), 1-20, 1992.

HALPERN, O. Beautiful data: A history of vision and reason since 1945 (experimental futures). Durham, NC: Duke University Press, 2014.

KAMENS, D. H. Globalization and the growth of international educational testing and national assessment. Comparative Education Review, 54(1), 5-25, 2009.

KAZAMIAS, A.; MASSIALAS, B. G. Comparative education. In H. E. Mitzel (Ed.), Encyclopedia of educational research (Vol 1). New York: Free Press, 1982.

KULLENBERG, C. The quantification of society: A study of a Swedish research institute and surveybased social science. Gothenburg: Gothenburg University, 2012.

LESKO, N. Act your age: A cultural construction of adolescence. New York \& London: Routledge Falmer, 2001.

LINDBLAD, S.; PETTERSSON, D.; POPKEWITZ, T. S. International comparisons of school results: A systematic review of research on large scale assessments in education. Stockholm: Swedish Research Council, 2015.

MEAD, A. G. H. Mind, self, and society from the standpoint of a social behaviorist. Chicago: Chicago University Press, 1934.

MOURSHED, M.; PATEL, J; SUDER, K. Education to Employment: Getting Europe's Youth into Work. McKinsey \& Company, 2014.

NOAH, H. J.; ECKSTEIN, M. A. Toward a science of comparative education. New York: Macmillan, 1969.

NOWOTNY, H.; SCOTT, P.; GIBBONS, M. Introduction: Mode 2 revisited: The new production of knowledge. Minerva, 41(3), 179-194, 2003.

OECD. Assessing scientific, reading and mathematical literacy: A framework for PISA 2006. Paris: OECD, 2006.

OECD. Education at a Glance 2015: OECD Indicators. OECD Publishing, 2015, doi: 10.1787/eag-2015em.

OECD. PISA 2015 results (Vol. III): Students’ well-being PISA. Paris: OECD Publishing, 2017.

PETTERSSON, D. The development of the IEA: The rise of large-scale testing. In. NORDIN, A; SUNDBERG, D. (Eds.), Transnational policy-flows in European education: Conceptualizing and 
governing knowledge. East Greenwich: Oxford Studies in Comparative Education. Symposium Books, p. 105-122, 2014.

POOVEY, M. A history of the modern fact: Problems of knowledge in the sciences of wealth and society. Chicago: University of Chicago Press, 1998.

POPKEWITZ, T. S. Paradigm and ideology in educational research: The social function of the intellectual. London \& New York: Routledge, 1984.

POPKEWITZ, T. S.; Lindblad, S. Education Governance and Social Integration and Exclusion: Studies in the Powers of Reason and the Reasons of Power.Uppsala Reports on Education; n. 39. Uppsala: Uppsala university, Department of Education, p. 377-420, 2001.

POPKEWITZ, T. S. Cosmopolitanism and the age of school reform: Science, education, and making society by making the child. New York: Routledge, 2008.

POPKEWITZ, T. S. The impracticality of practical research: A history of sciences of change that conserve. Ann Arbor: The University of Michigan Press, 2020.

POPKEWITZ, T. S.; Lindblad, S. Educational governance and social inclusion and exclusion: Some conceptual difficulties and problematics in policy and research. Discourse: Studies in the Cultural Politics of Education, 21(1), 5-44, 2010.

PORTER, T. M. Trust in numbers: The pursuit of objectivity in science and public life. Princeton, NJ: Princeton University Press, 1995.

PORTER, T. M. Funny numbers. Culture Unbound, 4, p. 585-598, 2012.

ROSE, N. Powers of freedom: Reframing political thought. Cambridge: Cambridge University Press, 1999.

ROSELLÓ, P. Marc-Antoine Jullien de Paris: Père de l'Education Comparée et Précurseur du Bureau International de l'Education. Geneva: International Bureau of Education, 1943.

SMITH, A. An inquiry into the nature and causes of the wealth of nations. London: Methuen \& Co. Ltd, 1776.

STEINER-KHAMSI, G.; WALDOW, F. World yearbook of education 2012: Policy borrowing and lending in education. Milton Park: Routledge, 2012.

TÖNNIES, F. Community and society (Gemeinschaft und Gesellschaft).New Brunswick, NJ \& London: Transaction Publishers, 2007.

\section{Correspondência}

Sverker Lindblad: É Professor em Educação e Educação Especial na Universidade de Gotemburgo, Suécia. A sua investigação incide sobre as interações entre educação e sociedade e entre organizações e processos educacionais. Trabalha nas áreas de investigação e de ensino em teoria do currículo, análise da política educativa, e organizações e processos escolares.

E-mail: sverker.lindblad@ped.gu.se

Orcid: 0000-0003-3481-3686

Daniel Pettersson: Trabalha atualmente na Universidade de Gävle e da Universidade de Uppsala, na Suécia, como Professor Associado. Seus atuais interesses de pesquisa versam as avaliações internacionais em larga escala, as narrativas políticas, educação comparada e a história da educação. Coeditou, muito recentemente, a obra New Practices of Comparison, Quantification and Expertise in Education. É atualmente editor-chefe do Nordic Journal of Studies in Educational Policy.

E-mail: daniel.pettersson@hig.se 
Thomas S. Popkewitz: É professor no Departamento de Currículo e Instrução da Universidade de Wisconsin-Madison, Estados Unidos. A sua investigação atual incide nas avaliações internacionais e na procura de conhecimento 'prático e útil' como práticas culturais na fabricação de tipos de pessoas. Os seus estudos cruzam as áreas dos estudos curriculares, a sociologia política da educação, e a história cultural - de modo a analisar as políticas de conhecimento educacional e o paradoxo da exclusão e abjeção nos esforços para a inclusão.

E-mail: tspopkew@wisc.edu

Orcid: 0000-0002-5058-2235

Texto publicado em Currículo sem Fronteiras com autorização dos autores 